\title{
Evolving Towards the Hypercycle: A Spatial Model of Molecular Evolution
}

\author{
Camille Stephan-Otto Attolini ${ }^{\text {a }}$, and Peter F. Stadler b,a \\ ${ }^{a}$ Institut für Theoretische Chemie und Molekulare Strukturbiologie, \\ Universität Wien, Währingerstraße 17, A-1090 Wien, Austria \\ \{camille,studla\}@tbi.univie.ac.at \\ ${ }^{\mathrm{b}}$ Lehrstuhl für Bioinformatik, Institut für Informatik, Universität Leipzig, \\ Kreuzstraße 7b, D-04103 Leipzig, Germany. \\ \{camille,studla\}@bioinf.uni-leipzig.de
}

\begin{abstract}
We extend earlier cellular automata models of spatially extended hypercycles by including an explicit genetic component into the model. This allows us to study the sequence evolution of hypercyclically coupled molecular replicators in addition to considering their population dynamics and spatial organization. In line with previous models, that considered either spatial organization or sequence evolution alone, we find both temporal oscillations of the relative concentration of the species forming the hypercycles as well as the formation of spatial organisations including spiral waves. We also confirm the greatly increased robustness of the spatially extended hypercycle against various classes of parasites.

We find the sequence evolution of each of the hypercyclically coupled populations proceeds (after an inital selection-dominated phase) in a drift-like manner that can be described by a diffusion process in sequence space. Kimura's theory of neutral evolution is therefore applicable on long time-scales despite the fact that the hypercycle exhibits extreme periodic changes in population sizes and that are governed solely by frequency-dependent selection.
\end{abstract}

Key words: Quasispecies, hypercycle, cellular automata, RNA secondary structure

\section{Introduction}

The RNA World hypothesis $[14,13]$ proposes a self-contained biochemical system preceeding the origin of modern cellular life-forms, in which RNA molecules act both as genetic material and as enzymes [37]. The possibility of an RNA World depends on the capability of the RNA molecules to catalyze the chemical reactions necessary to replicate RNAs [3]. This scenario is supported both by the wide range of catalytic activities that can be realized by relatively 
small ribozymes $[21,25,26,30,55]$, and by the usage of RNA catalysis at crucial points in modern cells $[24,7,35]$. Plausible ribozyme catalyzed pathways for a late-stage ribo-organism are discussed in [26], the role and evolution of co-enzymes in a putative RNA world is explored in [22]. While the templateinduced synthesis of oligonucleotides from smaller oligonucleotide precursors was successfully demonstrated in the laboratory $[56,57,44,58]$, it seems impossible to replicate longer sequences without an enzyme [37]. Approaches to engineering a ribozyme-replicase have been very promising $[23,25,38]$. These experiments show that self-replication is most likely within the catalytic repertoire of nucleic acids [34]. So far, however, they have not resulted in an RNA ribozyme that can catalyze its own replication with an efficiency that could have sustained a genetic system on the early Earth.

A central issue in models of prebiotic evolution is the integration of information that is necessary to bridge the gap between a simple system of replicating molecules and the complexity of a modern cell $[9,27]$. The template length is limited by the accuracy of the replication mechanism, which is necessarily error-prone due to mutations [8]. In principle the error threshold can be circumvented by evolving more accurate replicases that could be encoded by longer sequences $[40,39,52]$. Such a bootstrapping mechanism, however, requires a functional replicase-ribozyme to start with. By comparison with known ribozymes such a molecule would probably be about 100nt long, while the current limit for non-catalyzed replication is less than $20 \mathrm{nt}$.

An alternative mechanism that allows the accumulation of heritable information is the cooperation of self-replicators, introduced in the Hypercycle model [9]. It was soon noticed, however, that hypercycles and similar models are vulnerable to various kinds of parasites in homogeneous solution [33,5]. Not surprisingly, the number of coupled replicators increases only very slowly in models of self-replicators with mutation $[32,15]$.

Boerlijst and Hogeweg [4] and, later, Streissler [51] (in a PDE setting) and Cronhjort and Blomberg [6] showed that the problem of parasite invasion can be alleviated by considering spatially organized systems. Most theoretical studies have demonstrated that some kind of spatial structure is indispensable for the persistence and/or the parasite resistance of any feasible replicator system, see e.g. [53,1,59], although a chemical kinetics with product inhibition can have a similar effect in some parameter ranges $[46,48]$.

The shape of the fitness function, and more generally the accessibility of mutants from a given population, crucially influences the dynamics of evolution $[42,10,47]$. In the case of RNA it has been demonstrated that the genotypephenotype is dominated by so-called neutral networks that percolate through sequence space, thereby allowing efficient exploration by means of neutral drift confined to the neutral networks $[42,20,19]$. Recently, it was shown that a sim- 
ilar mechanism allows population of autocatalytic self-replicators to explore sequence space in a diffusion-like manner [45].

Simple finite population models of hypercycles have been considered e.g. in [2]. For larger, not necessarily hypercyclic, networks destabilization in homogeneous solution has been observed as a consequence of stochastic fluctuations [36]. The only study of sequence evolution of a hypercycle based on an explicit genotype-phenotype map is [11], which concentrates short cycles in a homogeneous medium. This study focusses on error-threshold phenomena similar to those described for uncatalyzed replicators $[12,20]$.

In this contribution we combine the macroscopic modelling of the spatiotemporal population dynamics of self-replicators with the microscopic modelling of the motion of populations of replicators in sequence space. To this end, replicating polymers are explicitly represented by their sequence in a CAlike universe. All reaction rates are derived from the (secondary) structures of the molecules which can be computed directly from their sequences. The parameters of the population dynamics are therefore not external ingredients of the simulation but intrinsic in the model itself [41]. In addition to demonstrating that we recover the typical dynamical features of simpler models of hypercyclic systems, we focus here on the dynamics in sequence space and show that Kimura's model of neutral evolution is applicable at least when time-scales are considered that are much larger than the oszillations of species in the population dynamics of a hypercycle.

\section{The model}

We consider a stochastic version of a second order replicator equation [43] with mutation, i.e., a replication mechanism of the form

$$
x+y \longrightarrow x+y+z
$$

The symbol $x$ represents the sequence of a template RNA molecule that, with the aid of the replicase ribozyme $y$, is copied to produce an RNA sequence $z$, which can be the same as the template, $x=x$, in the case of correct copying, or a mutant $z \neq x$. In addition we consider a slow uncatalyzed replication mechanism of the form $x \longrightarrow x+z$.

Each RNA sequence is interpreted as a self-replicator that also has the ability to catalyze the replication of other RNAs. Catalytic activities and replication rates are dependent on the molecules' secondary structure ${ }^{1}$. Secondary

\footnotetext{
1 A secondary structure $\mathcal{S}$ is a special type of contact structure, represented by a
} list of base pairs $[i, j]$ with $i<j$ on a sequence $x$, such that for any two base pairs 


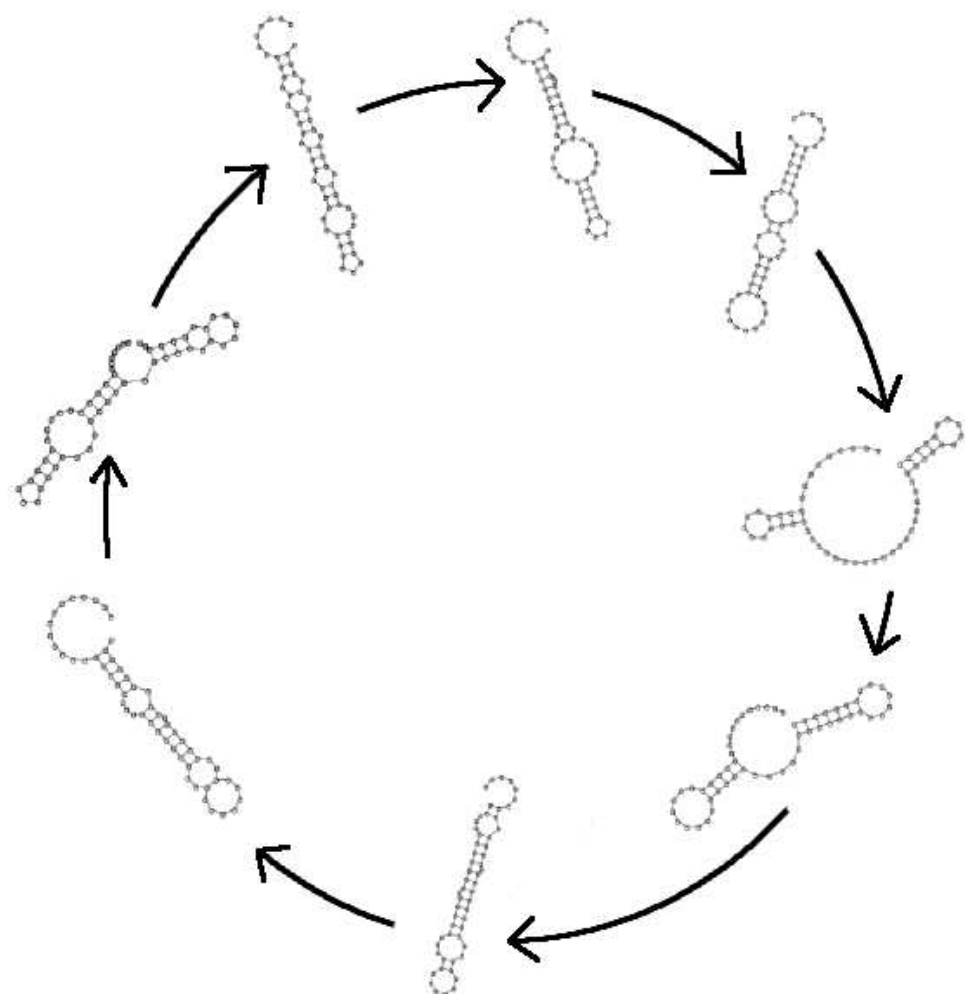

Fig. 1. The target set is a hypercycle with 8 members. All sequences have length $n=56$.

structures of RNA molecules can be computed efficiently by means of a dynamic programming approach [60] based on empiral parameters [31]. We use the Vienna RNA Package $[17,16]$ for this purpose. The optimal reaction rates are realized by the "perfect" target-hypercycle in Fig. 1. It is known that self-organization providing resistance to parasites is possible only in cycles of 6 or more members, while cycles of 3-5 members are quickly destroyed [18]. Therefore we choose an 8 members hypercycle for our model.

The interaction topology of our target set is a hypercycle with 8 members $\mathcal{T}_{1}$ through $\mathcal{T}_{8}$. The target structures $\mathcal{T}_{k}$ were picked at random. In order to investigate resistance against parasites, we consider selfish parasites and short-cut parasites besides the ordinary members of the hypercycle. All rates for parasite sequences are computed in the same way as for the target-set members. Indeed, technically, the parasites are treated as additional targetstructures.

For each sequence $x$ in a population $\mathbb{P}$ we compute its secondary structure $\mathcal{S}(x)$ using the Vienna RNA Package [17]. Then we determine its structure distance

$\overline{[i, j] \text { and }}[k, l]$ with $i \leq k$ holds: $(i) i=k$ if and only if $j=l$, and (ii) $k<j$ implies $i<k<l<j$. 
$D\left(\mathcal{T}_{k}, \mathcal{S}(x)\right)$ to the target shapes $\mathcal{T}_{k}$. For simplicity we define $D(\mathcal{X}, \mathcal{Y})$ as the number of base-pairs that $\mathcal{X}$ and $\mathcal{Y}$ do not share. Finally, we assign $\mathcal{S}(x)$ to the hypercycle-member $h$ that minimizes the distance $D\left(\mathcal{T}_{k}, \mathcal{S}(x)\right)$. We write $\mathbb{P}^{h}$ for this sub-population of sequences whose structure ist closest to the target shape $\mathcal{T}_{h}$.

Once the group $h$ has been determined for every sequence the replicationdecay-catalysis process in simulated as outlined in [4], Fig. 2:

Decay: Sequence $x$ has a decay probability that depends linearly on the distance to the target structure:

$\delta_{x}=1+D\left(\mathcal{T}_{k}, \mathcal{S}(x)\right)$

Replication: Sequence $x$ has a probability to self-replicate without the help of a catalyst that depends inversely on the distance to the target structure:

$\alpha_{x} \sim \frac{1}{1+D\left(\mathcal{T}_{k}, \mathcal{S}(x)\right)}$

Catalyzed Replication: When a self-replicator has neighbors that correspond to their catalysts in the direction of the reaction, the probability (rate) of catalysis is largely improved. As well as self-replication rates depends on fitness, also the performance of catalysts is defined by their distance to the target. The similar a phenotype is to the corresponding target, the better its rate as catalyst will be. The total replication rate is therefore

$$
\rho_{x}=\frac{1}{1+D\left(\mathcal{T}_{k}, \mathcal{S}(x)\right)}+\sum_{y \text { catalyzes x }} \frac{C}{1+D\left(\mathcal{T}_{y}, \mathcal{S}(y)\right)}
$$

where $C=8000$ is the relative rate of catalyzed versus uncatalyzed replication.

Mutations occur as errors during replication. As in Eigen's quasispecies model [8] we assume a uniform per-nucleotide rate $p$ of incorporating an erroneous letter. These point mutations of the parental sequence $x$ have a high probability of changing the secondary structure. Since these structural changes may be large [42] we have significant probability that a mutant sequence will belong to either a different class of hypercycle members or to one of the parasite classes.

The population $\mathbb{P}$ of replicators is spread out on a 2-dimensional grid with periodic boundary conditions, typically consisting of $200 \times 200$ cells. In this respect our simulation resembles those described in $[4,6]$. Each cell can be empty or occupied by a RNA single sequence. Diffusion is modeled using the Toffoli-Margolus scheme [54]. We use two diffusion steps within each simulation time step. Simulations are initialized by randomly placing 200 to 1000 initial sequences on the grid. The sequence in an occupied cell dies with a rate proportional to $\delta_{x}$. For every empty cell we compute the replication rates $\rho_{z}$ for all its neighboring cells, assuming that the replication of $z$ is catalyzed only by those neighbors that correspond to the preceding class in the hypercycle topology, Fig. 2. According to the model presented in [4], we consider possible catalysts only in the direction of the replication. The sequence with the largest values of $\rho_{z}$ invades the empty cell. Cells are chosen for update in 


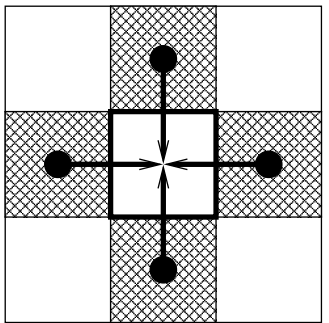

Possible Replicators

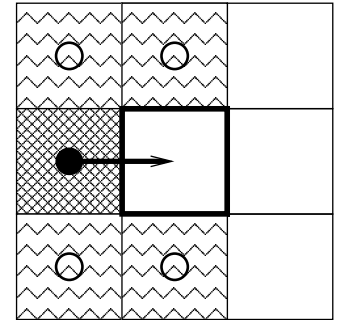

$\widetilde{M}$ Possible Catalysts

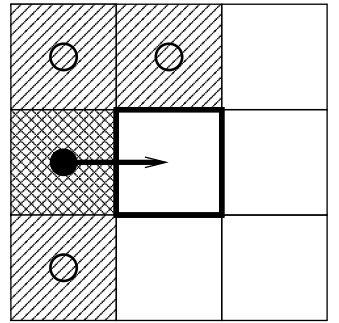

Actual Catalysts

Fig. 2. Rules of replication. For each of the neighbors $(\bullet)$ of the empty cell (marked by a bold outline) the replication rate $\rho_{z}$ is computed taking into account their neighbors in the direction of the replication (o) as potential catalysts. The neighbor with the largest values of $\rho_{z}$ invades the empty position. In this example, for the chosen replicator, only three of its neighbours are catalysts according to the hypercycle topology.

random order until every occupied cell has been updated..

Several variables are measured througout the simulations: the number $N_{k}=$ $\left|\mathbb{P}^{k}\right|$ of individuals per group, the average distance $\bar{D}$ to target over the whole system and over each group, the diversity $\theta_{k}$ between individuals in a class of replicators and number $Y_{k}$ of different sequences belonging to target class $k$. The diversity of a group is computed as proposed in [45]

$$
\theta_{k}=\frac{1}{N_{k}\left(N_{k}-1\right)} \sum_{x \neq y \in \mathbb{P}^{k}} d_{H}(x, y)
$$

where $d_{H}(x, y)$ is the Hamming distance of the sequences $x$ and $y$. In [45] it is shown that replicators with interactions tend to minimize diversity until they end in a quasispecies-like distribution.

\section{Simulation Results}

\subsection{Spatial Pattern Formation}

We first consider a universe without parasites, i.e., all sequences are assigned to one of the structures of the hypercycle-members. As in [4] we observe spiral waves when every member of the cycle has a minimum concentration. In almost every run, a first period of disorder is followed by the birth of a spiral which contains sequences of every group, ordered depending on the topology of the targets. It is important to notice that without a minimum fitness, individuals 


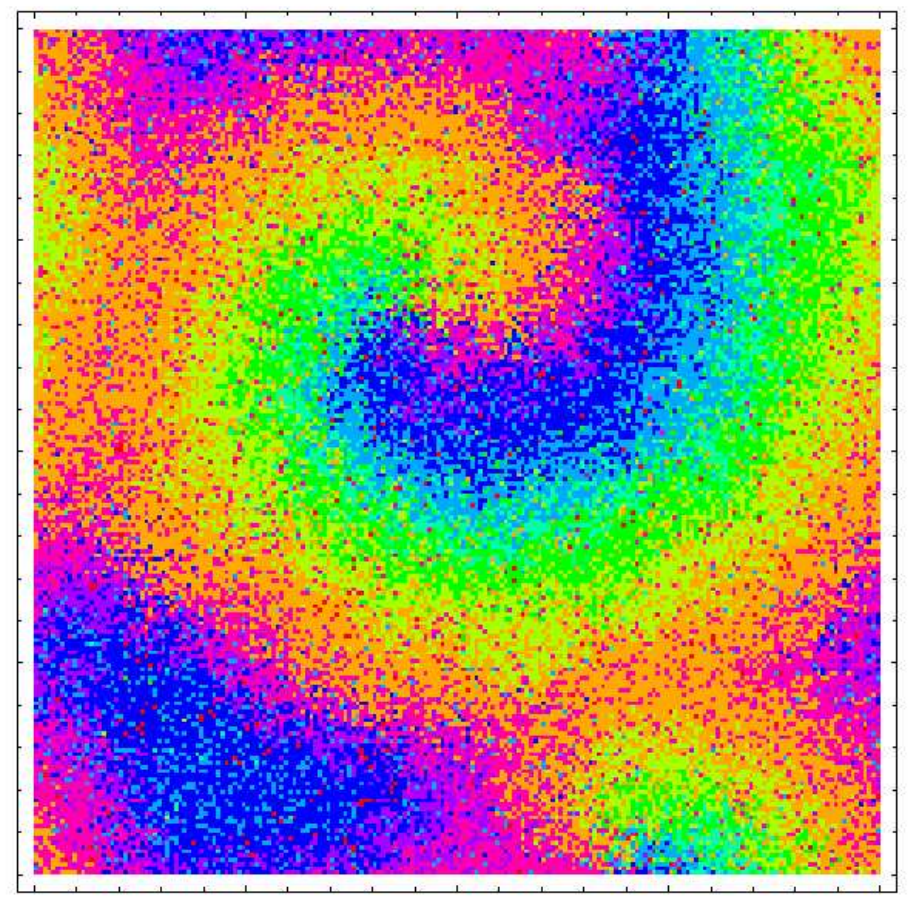

Fig. 3. Spirals formed after 3000 generations in an evolution experiment started with 300 random sequences in the absence of parasites.

Simulation parameters: grid size $L \times L=200 \times 200$, sequence length $n=56$, mutation rate $p=3.5 \times 10^{-4}, 2$ diffusion steps between replication steps. Simulation parameters are the same in all figure unless explicitly stated otherwise.

of a group would die before they could get any help to replicate, so that evolved enough sequences of every group must be present in order to the spatial patterns to emerge. Once the spiral is formed, the sequences continue approaching the target but in a much slower pace, in fact, in some simulations we observed an oscillatory behavior of the fitness average depending on the number of sequences present in the system at any given moment.

Some groups could reach the target, while others may stay away without breaking the dynamics. In the case where one group will get to the target while the others had a poor fitness, however, the system sometimes collapses to the survival of only the single fittest species. This is only possible when the group which is catalyzed by this "master species" is not present in the system: since the rate of catalysis depends also on the fitness, so that the "follower" will increase its concentration at the expense of the "master species".

When all members of the cycle are present with a minimum concentration and fitness, a change of behavior occurs and an oscillatory behavior in the number $N_{k}$ of sequences per group is observed, see Fig. 4 . The amplitude of this waves 


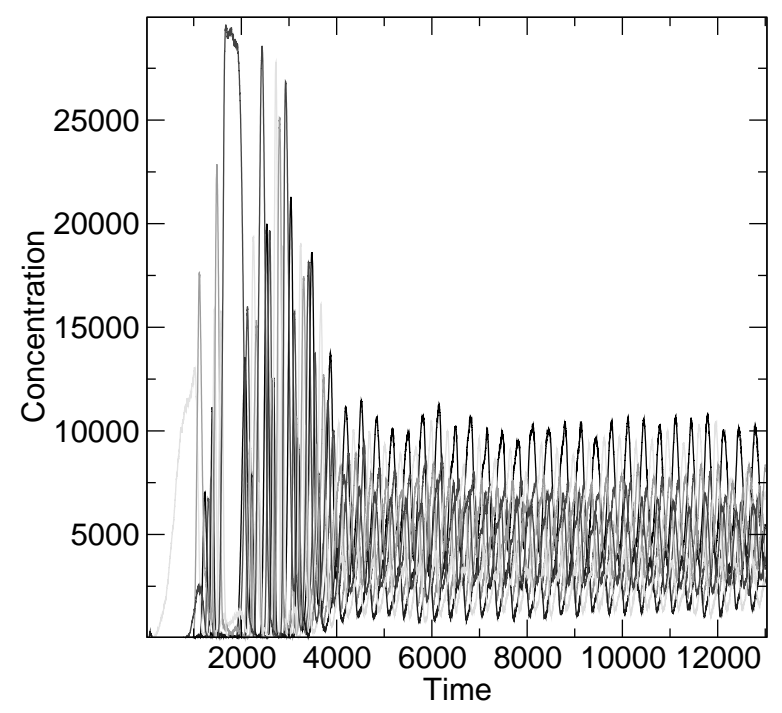

Fig. 4. Evolution towards the target hypercycle. After a transient period of disorder, the concentrations $Y_{k}$ of the individual member-classes of the hypercycle (different colors) exhibit regular oscillations.

depends on the ratio between self-replication and catalyzed replication rates. If this ratio is too large, the abundance of sequences of one group will lead to a very fast growth of the next group in the hypercycle, giving almost no space for other members to replicate. Only one or two groups fill the entire lattice at any given point in time, making it more difficult or impossible to create the spirals.

When a selfish parasite is introduced, a first period is observed where members of the hypercycle, as well as the parasite, appear and disappear from the system without much order. For some time both parasite and hypercycle can coexist, but it ends in the parasite being expulsed from the system and the spirals arise. Of course, mutations from regular sequences may jump to the parasite group, implying that the parasite has members almost all the time without being harmful for the system. These parasitic sequences typically are erradicated before they can evolve towards high replication rates. The spirals in this case are not as regular as those without parasite, nevertheless they are stable and can coexist with an invading parasite.

The case of the short-cut parasite is quite similar. The system is stable against this kind of parasite and only a few times the runs ended with the shorter cycle formed in this topology. In the majority of the simulations, however, the parasite was expelled from system after some time. The reason for this increased 


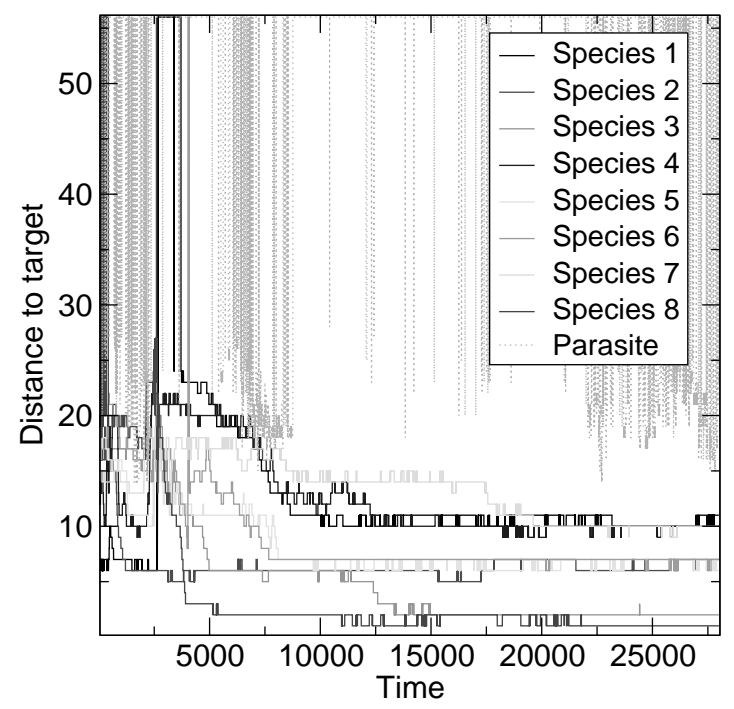

Fig. 5. Evolution of the mean fitness of the individual classes (different colors). After a period of disorder, the parasite (gray curve in the upper part of the plot) in unable to re-invade the system. It dies our before it can reach sequences that are near-optimal parasites (distance 0 to target).

resistance appears to lie in the the genotype-phenotype map derived from the RNA folding algorithm. The fact that fitness depends on the secondary structure gives allows the hypercycle to evolve towards a stronger configuration while the parasite is left behind: from the fitness plot one can see how for some period the parasite evolves more or less the same way as the other members of the hypercycle. Nevertheless, everytime the parasite is expelled from the system, it looses the fitness it could have won before, becoming a much weaker species. It is clear that stability of the hypercycle is due not only to the spatial configuration but also to the advantage of its members in an evolutionary way.

\subsection{Population Structure}

Diversity depends strongly on the initial conditions, in particular on the number of sequences first introduced to the system. To make replicators evolve towards the targets, it is important to keep a high selectivity among them, this in turn can make it harder for the system to reach the desired organization. Starting with less than 100 sequences leads, almost all the time, to the death of all species or the survival of one. If selection is lowered it is possible 


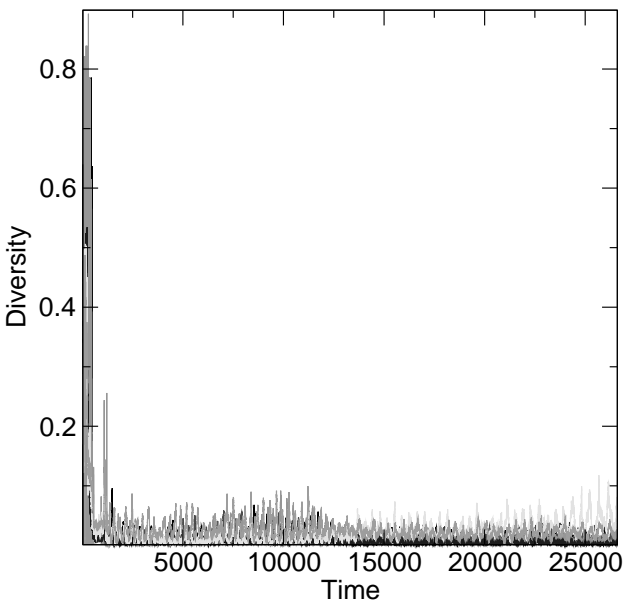

(a)

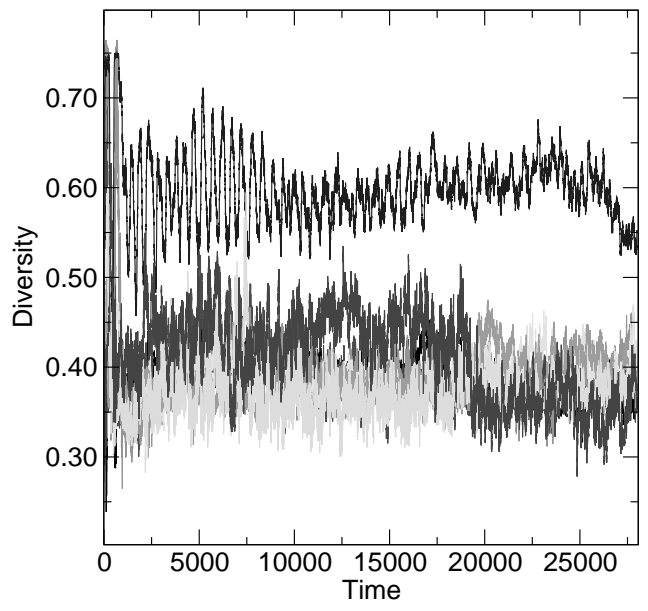

(b)

Fig. 6. Diversity of the system with different initial conditions. Simulation parameters for both plots are the same except for the number of initial sequences in the lattice. For figure (a) 300 sequences were used while in figure (b) 800 individuals started the simulation. It can be seen that when the number of species at the begining is high enough, diversity is kept high until the end.

to start with one sequence but evolution towards the targets will be slower. In most cases, starting with 200 or 300 sequences allows the system to survive even with higher selection rates. In this case, diversity falls very quickly to almost zero and is maintained like that until the end of the simulation, see Fig. 6(a).

When the number of initial sequences is increased, diversity stays higher and oscillates depending on the number of species per group, Fig. 6(b). This means that if diversity is high enough at the beginning, then it is maintained by the system. We believe that this is due to the interactions and catalyzed replications: selection on a single member becomes less important when its replication is improved by the others. Even a fast dying sequence can stay in the lattice because of its even faster catalyzed replication.

A quasispecies-like behavior is observed if diversity is low. The distribution of the number of individuals with the same sequence is centered around a "master sequence" in each class of hypercycle-members; a large fraction of the populations consists of individuals that occur only in a single copy. These "explorers" of the sequence space are lost and replaced by others within a few generations, Fig. 7. 


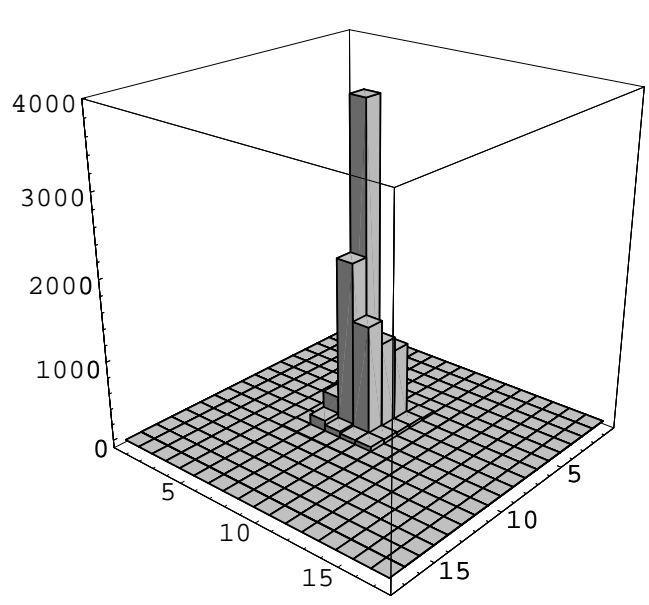

(a)

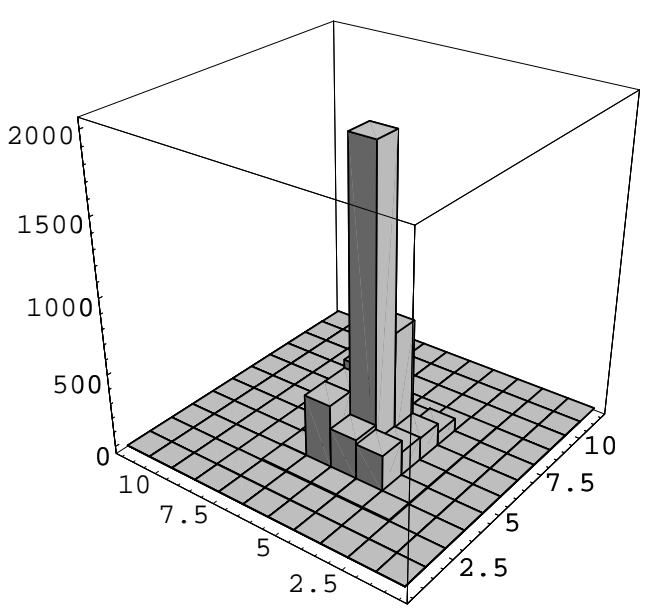

(b)

Fig. 7. Distribution of sequences in each class of hypercycle members. Only a few sequences are present with almost all the individuals of the group while the rest of sequences are represented by only one individual. Figures (a) and (b) show the distribution of two different members in the same simulation.

\subsection{Drift and Diffusion in Sequence Space}

The profile of the class $k$ of the hypercycle at time $t$ is the $4 \times n$ vector $\mathbf{p}^{k}(t)$ whose components are the frequencies of the 4 types of nucleotides at each sequence position [45]. The overall movement of the population in sequence space can be quantified in terms of the correlation function

$$
g(\tau)=\frac{1}{T_{2}-T_{1}+1} \sum_{t=T_{1}}^{T_{2}}\|\mathbf{p}(t+\tau)-\mathbf{p}(t)\|^{2}
$$

computed for suitable intervals of measurement $\left[T_{1}, T_{2}\right]$. The mobility of the population in sequence space is conveniently quantified in terms of the diffusion constant $D$ which is defined as the slope of $g(\tau) \sim D \tau$.

As expected from simulations both of RNA based quasispecies [20] and from a simple model of interacting molecular replicators [45] we observe a linear dependence of the diffusion constant on the per-site mutation rate $p$, see Fig. 8b. We should expect that small differences in the diffusion constants of different sub-populations should exist since the diffusion constant should depend on the fraction $\nu$ of mutations that do not change the secondary structure. It is known that $\nu$ depends on the secondary structure in question [20]. We have not been able to detect significant difference in the diffusion constants of individual sub-populations (data not shown) since the effects are small and 


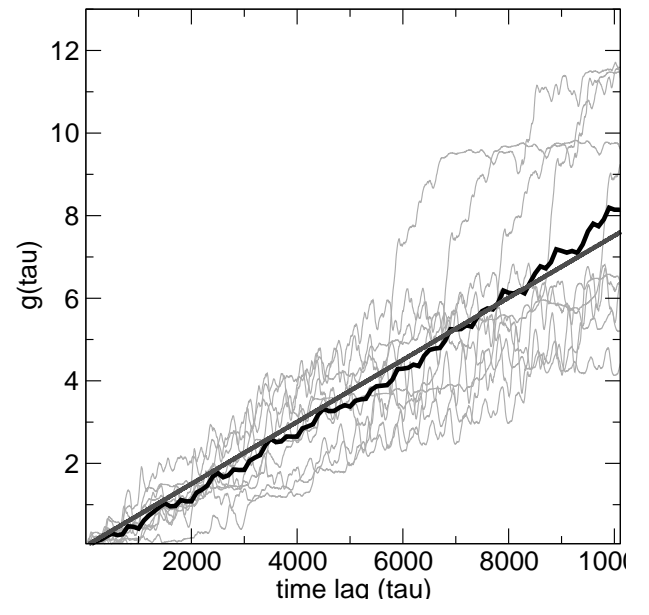

(a)

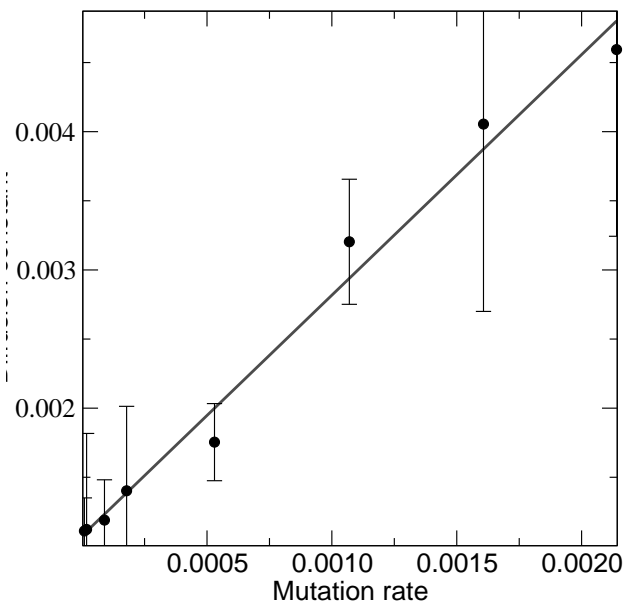

(b)

Fig. 8. Displacement of the profile and diffusion constant $D$ vs mutation rate. (a) The Displacement of the profile with time, $g(\tau)$, is shown in gray for individual sub-populations $\mathbb{P}^{k}$. The average of $g(\tau)$ over all sub-populations is displayed as bold black line. (b) The slope of $g(\tau)$ defines the diffusion constant in sequence space. As expected, there is a linear dependence between diffusion constant $D$ and single digit mutatation frequency $d$. The data are averaged over 16 different runs and all species.

would require much more extensive simulations in order to obtain sufficiently accurate estimates of $D$ for each species separately.

\section{Discussion}

We have simulated here a simple hypercyclic network that incorporates strong interactions between species and hence a complicated population dynamics, spatial organization, and an explicit representation in sequence space. Our first main conclusion is that the behavior of such an integrated computer simulation is consistent with earlier findings on both the population dynamics (such as the existence of limit cycles) of hypercycles and on the effects of considering a spatially extended system (such as the formation of spiral waves and resistance against various types of parasites). The resistance of the system against shortcut parasites in addition to "dead-end" parasites is a very important result since it shows that spatially extended hypercycles are indeed evolutionarily very stable systems. This is in sharp contrast to hypercycles in homogeneous solution $[9,5,49,50]$.

Furthermore, we demonstrate here a mode of sequence evolution that is domi- 
nated by drift and hence can be described in terms of Kimura's Neutral theory $[28,29]$. This does not mean, of course, that selection does not play a role: the exclusion of parasites, the internal dynamics of the population, as well as the sequence-evolution in the initial phase of the simulation are clearly dominated by selection.

Changing any of the initial conditions, parameters, target structures, or just the random numbers used to model the mutation events, of course leads to very different sequences. Nevertheless, the main characteristics of the system are robust and differ only in small details from one set of conditions to the other. We therefore conclude that the ability of such an RNA based system to evolve towards a robust spatially extended organization is intrinsic to autocatalytic self-replicating molecules as soon as the sequence-structure relationship is dominated by extensive neutral networks, as is the case for RNA.

\section{Acknowledgments}

This work was supported in part by Consejo Nacional de Ciencia y Tecnología, CONACyT, and the DFG Bioinformatic Initiative BIZ-6/1-2.

\section{References}

[1] S. Altmeyer and J. S. McCaskill. Error threshold for spatially resolved evolution in the quasispecies model. Phys. Rev. Lett., 86:5819-5822, 2001.

[2] M. A. Andrade, A. J. Garcia-Tejedor, and F. Montero. Study of an error-prone hypercycle formed from two kinetically distinguishable species. Biophys. Chem., 40:43-57, 1991.

[3] D. P. Bartel and P. J. Unrau. Constructing an rna world. Trends Cell Biol., 9(12):M9-M13, 1999.

[4] M. C. Boerlijst and P. Hogeweg. Spiral wave structure in pre-biotic evolution: hypercycles stable against parasites. Physica D, 48:17-28, 1991.

[5] C. Bresch, U. Niesert, and D. Harnasch. Hypercycles, parasites, and packages. J. Theor. Biol., 85:399-405, 1980.

[6] M. B. Cronhjort and C. Blomberg. Hypercycles versus parasites in a two dimensional partial differential equations model. J. Theor. Biol., 169:31-49, 1994.

[7] J. A. Doudna and T. R. Cech. The chemical repertoire of natural ribozymes. Nature, 418:222-228, 2002. 
[8] M. Eigen. Selforganization of matter and the evolution of biological macromolecules. Naturwissenschaften, 58:465-523, 1971.

[9] M. Eigen and P. Schuster. The Hypercycle. Springer-Verlag, New York, Berlin, 1979.

[10] W. Fontana and P. Schuster. Continuity in evolution: On the nature of transitions. Science, 280:1451-1455, 1998.

[11] C. V. Forst. Molecular evolution of catalysis. J. Theor. Biol., 205:409-431, 2000.

[12] C. V. Forst, C. M. Reidys, and J. Weber. Evolutionary dynamics and optimization: Neutral Networks as model-landscape for RNA secondarystructure folding-landscapes. In F. Morán, A. Moreno, J. Merelo, and P. Chacón, editors, Advances in Artificial Life, volume 929 of Lecture Notes in Artificial Intelligence, pages 128-147, Berlin, Heidelberg, New York, 1995. ECAL '95, Springer.

[13] R. F. Gesteland and J. F. Atkins, editors. The RNA World. Cold Spring Harbor Laboratory Press, Plainview, NY, 1993.

[14] W. Gilbert. The RNA world. Nature, 319:618, 1986.

[15] R. Happel and P. F. Stadler. The evolution of diversity in replicator networks. J. Theor. Biol., 195:329-338, 1998.

[16] I. L. Hofacker. Vienna RNA secondary structure server. Nucl. Acids Res., 31:3429-3431, 2003.

[17] I. L. Hofacker, W. Fontana, P. F. Stadler, S. Bonhoeffer, M. Tacker, and P. Schuster. Fast folding and comparison of RNA secondary structures. Monatsh. Chemie, 125(2):167-188, 1994.

[18] P. Hogeweg and N. Takeuchi. Multilevel selection in models of prebiotic evolution: Compartments and spatial self-organization. Origins of Life and Evolution of the Biosphere, 33:375-403, 2003.

[19] M. A. Huynen. Exploring phenotype space through neutral evolution. J. Mol. Evol., 43:165-169, 1996.

[20] M. A. Huynen, P. F. Stadler, and W. Fontana. Smoothness within ruggedness: the role of neutrality in adaptation. Proc. Natl. Acad. Sci. (USA), 93:397-401, 1996. SFI preprint 95-01-006, LAUR-94-3763.

[21] M. Illangasekare and M. Yarus. A tiny RNA that catalyzes both aminoacylRNA and peptidyl-RNA synthesis. RNA, 5:1482-1489, 1999.

[22] V. R. Jadhav and M. Yarus. Coenzymes as coribozymes. Biochimie, 84:877-888, 2002.

[23] K. D. James and A. D. Ellington. The fidelity of template-directed oligonucleotide ligation and the inevitability of polymerase function. Orig. Life Evol. Biosph., 29:375-390, 1999. 
[24] D. C. Jeffares, A. M. Poole, and D. Penny. Relics from the RNA world. J. Mol. Evol., 46:18-36, 1998.

[25] W. K. Johnston, P. J. Unrau, M. J. Lawrence, M. E. Glasner, and D. P. Bartel. RNA-catalyzed RNA polymerization: Accurate and general RNA-templated primer extension. Science, 292:1319-1325, 2001.

[26] G. F. Joyce. The antiquity of RNA-based evolution. Nature, 418:214-221, 2002.

[27] S. A. Kauffman. The Origin of Order. Oxford University Press, New York, Oxford, 1993.

[28] M. Kimura. Solution of a process of random genetic drift with a continuous model. Proc. Natl. Acad. Sci. USA, 41:144-150, 1955.

[29] M. Kimura. The Neutral Theory of Molecular Evolution. Cambridge University Press, Cambridge, UK, 1983.

[30] N. Lee, Y. Bessho, K. Wei, J. W. Szostak, and H. Suga. Ribozyme-catalyzed tRNA aminoacylation. Nat. Struct. Biol, 7:28-33, 2000.

[31] D. Mathews, J. Sabina, M. Zuker, and H. Turner. Expanded sequence dependence of thermodynamic parameters provides robust prediction of RNA secondary structure. J. Mol. Biol., 288:911-940, 1999.

[32] R. M. May and M. A. Nowak. Superinfection, metapopulation dynamics, and the evolution of diversity. J. Theor. Biol., 170:95-114, 1994.

[33] J. Maynard Smith. Hypercycles and the origin of life. Nature, 280:445-446, 1979.

[34] K. E. McGinness and G. F. Joyce. In search of an RNA replicase ribozyme. Chem. Biol., 10:5-14, 2003.

[35] P. B. Moore and T. A. Steitz. The involvement of RNA in ribosome function. Nature, 418:229-235, 2002.

[36] J. C. Nuñ and P. Tarazona. Lifetimes of small catalytic networks. Bull. Math. Biol., 56:875-898, 1994.

[37] L. E. Orgel. The origin of life - a review of facts and speculations. Trends Biochem. Sci., 23(12):491-495, 1998.

[38] N. Paul and G. F. Joyce. A self-replicating ligase ribozyme. Proc. Natl. Acad. Sci. USA, 99:12733-12740, 2003.

[39] A. Poole, D. Jeffares, and D. Penny. Early evolution: prokaryotes, the new kids on the block. Bioessays, 21:880-889, 1999.

[40] I. Scheuring, T. Czaran, P. Szabo, G. Karyoli, and Z. Toroczkai. Spatial models of prebiotic evolution: Soup before pizza? Origins of life, 33(4-5):329-355, 2003.

[41] P. Schuster. Evolution at molecular resolution. In L. Matsson, editor, Nonlinear Cooperative Phenomena in Biological Systems, pages 86-112. World Scientific, 1998. 
[42] P. Schuster, W. Fontana, P. F. Stadler, and I. L. Hofacker. From sequences to shapes and back: A case study in RNA secondary structures. Proc. Roy. Soc. Lond., B225:279-284, 1994.

[43] P. Schuster and K. Sigmund. Replicator dynamics. J. Theor. Biol., 100:533-538, 1983.

[44] D. Sievers and G. von Kiedrowski. Self-replication of complementary nucleotidebased oligomers. Nature, 369:221-224, 1994.

[45] B. M. R. Stadler. Diffusion of a population of interacting replicators in sequence space. Adv. Complex Systems, 5(4):457-461, 2002.

[46] B. M. R. Stadler, P. F. Stadler, and P. Schuster. Dynamics of autocatalytic replicator networks based on higher order ligation reactions. Bull. Math. Biol., 62:1061-1086, 2000.

[47] B. M. R. Stadler, P. F. Stadler, G. Wagner, and W. Fontana. The topology of the possible: Formal spaces underlying patterns of evolutionary change. $J$. Theor. Biol., 213:241-274, 2001.

[48] B. M. R. Stadler, P. F. Stadler, and P. R. Wills. Evolution in systems of ligation-based replicators. Z. Phys. Chem., 21-33:216, 2001.

[49] P. F. Stadler and R. Happel. The probability for permanence. Math. Biosc., 113:25-50, 1993.

[50] P. F. Stadler and P. Schuster. Permanence of sparse autocatalytic networks. Math. Biosc., 131:111-134, 1996.

[51] C. Streissler. Autocatalytic Networks Under Diffusion. PhD thesis, University of Vienna, 1992.

[52] P. Szabó, I. Scheuring, T. Czaran, and E. Szathmáry. In silico simulations reveal that replicators with limited dispersal evolve towards higher efficiency and fidelity. Nature, 420:278-279, 2002.

[53] V. Tereshko. Selection and coexistence by reaction-diffusion dynamics in fitness landscapes. Phys. Let. A, 260:522-527, 1999.

[54] T. Toffoli and N. Margolus. Cellular Automata Machines: A New Environment for Modeling. MIT Press, 1987.

[55] P. J. Unrau and D. P. Bartel. RNA-catalysed nucleotide synthesis. Nature, 395:260-263, 1998.

[56] G. von Kiedrowski. A self-replicating hexadeoxynucleotide. Angew. Chem. Int. Ed. Engl., 25:932-935, 1986.

[57] G. von Kiedrowski, B. Wlotzka, and J. Helbing. Sequence dependence of template-directed syntheses of hexadeoxynucleotide derivatives with 3'-5' pyrophosphate linkage. J. Angew. Chem. Int. Edn. engl., 28:1235-1237, 1989. 
[58] B. Wlotzka and J. S. McCaskill. A molecular predator and its prey: Coupled isothermal amplification of nucleic acids. Chemistry \& Biology, 4:25-33, 1997.

[59] E. Zintzaras, M. Santos, and E. Szathmáry. "Living" under the challenge of information decay: The stochastic corrector model vs. hypercycles. J. Theor. Biol., 217:167-181, 2002.

[60] M. Zuker and D. Sankoff. RNA secondary structures and their prediction. Bull. Math. Biol., 46(4):591-621, 1984. 